\title{
Perfil de emprendimiento de los estudiantes de la Universidad de Sonora, Unidad Norte de México
}

\section{Entrepreneurship profile of the students of the University of Sonora, Northern Unit of Mexico}

DOI: $10.46932 / \mathrm{sfjdv2n2-149}$

Received in: March 1st, 2021

Accepted in: May 30th, 2021

\author{
Jesús Martín Cadena-Badilla \\ Doctor en Planeación Estratégica y Dirección de Tecnología. Maestro Investigador Titular C. \\ Universidad de Sonora, México. \\ E-mail:mcadena@guaymas.uson.mx \\ Rafael Hernández León \\ Doctor en Planeación Estratégica y Dirección de Tecnología. Maestro Investigador Titular A. \\ Universidad de Sonora, México. \\ E-mail: rafael.hernandez@unison.mx
}

Joaquín Vásquez Quiroga

Maestro en Ciencias en Ingeniería Industrial. Estudiante de Doctorado. Maestro Investigador Titular A.

Universidad de Sonora, México.

E-mail: joaquin.vasquez@unison.mx

Francisco Javier León Moreno

Doctor en Planeación Estratégica y Dirección de Tecnología. Maestro Investigador Titular A.

Universidad de Sonora, México.

E-mail: javier.leon@unison.mx

Alfredo Heredia Bustamante

Doctor en Ciencias Administrativas. Maestro Investigador Titular A. Universidad de Sonora, México. E-mail: alfredo.heredia@unison.mx

\section{RESUMEN}

Actualmente, la mayoría de las instituciones de educación superior (IES), no cuenta con un perfil que permita el desarrollo de las capacidades de emprendimiento que necesita el profesional egresado. La actividad emprendedora es uno de los principales motores de desarrollo económico, principalmente por su función en la creación de empleo y en la expansión de sectores económicos; por otra parte, es un área del conocimiento aún nueva en el marco global de la investigación, y como tal, se encuentra en proceso para consolidar un escenario conceptual, lo que hace pertinente esta propuesta. El enfoque metodológico incluye una revisión del estado del conocimiento del tema, la aplicación de una escala de medición (Perfil e20), el análisis mediante métodos estadísticos multivariantes (Análisis de Factores), y la validación y fiabilidad de la escala usada. Se espera identificar las dimensiones y variables que caracterizan el perfil de emprendimiento de los estudiantes de la Unidad Norte de la Universidad de Sonora, el cual facilitaría la toma de decisiones en el diseño de estrategias curriculares y de enseñanza-aprendizaje.

Palabras Clave: Emprendedurismo, Instrumento Escala de Auto-Eficacia Emprendedora, Perfil de Capacidad Emprendedora. 


\begin{abstract}
Currently, most institutions of higher education (HEI) do not have a profile that allows the development of entrepreneurial skills needed by the graduate. Entrepreneurship is one of the main engines of economic development, mainly because of its role in job creation and the expansion of economic sectors; on the other hand, it is an area of knowledge still new in the global research framework, and as such, it is in the process of consolidating a conceptual scenario, which makes this proposal relevant. The methodological approach includes a review of the state of knowledge of the subject, the application of a measurement scale (Profile e20), the analysis using multivariate statistical methods (Factor Analysis), and the validation and reliability of the scale used. It is expected to identify the dimensions and variables that characterize the entrepreneurship profile of the students of Northern Unit of the University of Sonora, which would facilitate decision making in the design of curricular and teaching-learning strategies.
\end{abstract}

Key Words: Entrepreneurship, Instrument Scale of Entrepreneurship Self-Efficiency, Profile of Entrepreneurship.

\title{
1 INTRODUCCIÓN.
}

El emprendimiento ha sido estudiado a través de los años desde el punto de vista tanto de la economía como de la psicología, la sociología y la antropología; desarrollando aportes que se encargan del estudio del fenómeno desde un punto de vista social, sobre el cual existen relativamente pocos acuerdos (Guzmán y Trujillo, 2008). Se ha relacionado el emprendimiento con el descubrimiento de oportunidades rentables, concluyendo que las oportunidades de emprendimiento son aquellas situaciones en las que se puede introducir al mercado los métodos, bienes y servicios a un precio mayor que sus costos de producción (Shane y Venkataraman, 2000).

Se considera que los emprendedores contribuyen con los procesos en donde una persona transforma recursos, materiales y trabajo, asumiendo riesgos y por ende generando riqueza a través de las utilidades que genera la nueva organización productiva, (Hisrich, Langan-Fox \& Grant, 2007).

Por ello la iniciativa empresarial la define Hisrich como el "Proceso de crear algo nuevo con valor, dedicando el tiempo y el esfuerzo necesarios, asumiendo los correspondientes riesgos financieros, psicológicos y sociales, y obteniendo las recompensas resultantes de satisfacción e independencia económica y personal" (Hisrich, Peters, \& Shepherd, 2005) lo mencionado anteriormente refleja que las personas requieren de mucha decisión para asumir los riesgos que implica sacrificar los recursos financieros, materiales y humanos para generar una nueva empresa.

Las personas que deciden emprender tienen determinadas características o conductas que los caracterizan, “algunas de estas características pueden ser capacidad de planeación (fijar metas), manejo de contactos, comunicación efectiva, búsqueda de información, creatividad, trabajo en equipo, toma de decisiones y liderazgo" (Leiva Bonilla, 2008). Las condiciones de cada uno de los emprendedores es diferente y es por ello que cada uno tiene diferentes motivaciones para decidir emprender por necesidad 
o por oportunidad y según "un emprendedor por necesidad es aquel que se encuentra inmerso en el proceso emprendedor por no tener una mejor opción en el mercado laboral; mientras que un emprendedor por oportunidad es aquel que escoge crear una empresa basándose en la percepción de que existe una oportunidad de negocio no aprovechada -o aprovechada de forma incompleta- por las empresas existentes", (Fernández, 2015); aunque se debe considerar que en ocasiones los que emprenden por necesidad, tienden a abandonar su empresa cuando logran conseguir un empleo con ingresos fijos.

La Organización de las Naciones Unidas (ONU, 2016), plantea promover políticas orientadas al desarrollo que apoyen las actividades productivas, la creación de puestos de trabajo decentes, el emprendimiento, la creatividad y la innovación, y fomentar la formalización y el crecimiento de las microempresas y las pequeñas y medianas empresas, incluso mediante el acceso a servicios financieros.

\subsection{EMPRENDIMIENTO UNIVERSITARIO.}

El emprendimiento ha sido abordado desde la investigación universitaria desde múltiples enfoques. Durante los últimos años varios investigadores se han hecho interrogantes sobre la orientación y características de la investigación en emprendimiento, como una nueva área del conocimiento que poco a poco cobra mayor importancia en el concierto académico a nivel mundial (Matiz, 2009). En este sentido, se destacan los trabajos de Veciana (1999), Castillo (1999), Bruyat y Julien (2000), Low (2001), Busenitz et al. (2003), Cooper \& Schindler (2003), Moriano, Sánchez y Palací (2004), Zahra et al. (2006), López (2008), Martínez (2008), Cardozo (2010), Wiklund, Davidsson, Audretsch, \& Karlsson (2011), Nicolás y Rubio (2012), Herrera y Montoya (2013), Hidalgo, Kamiya y Reyes (2014), Karmarkar, Chabra y Deshpande (2014); y más recientemente, Murillo y Santillán (2015), Rodríguez (2015), Leitch y Volery (2017), entre muchos otros.

En cuanto al enfoque cuantitativo, más hacia la generación de medidas del emprendimiento, se destacan los trabajos de De Noble, Jung y Ehrlich (1999), Ehrlich, De Noble y Singh (2005), Sánchez, Lanero y Yurrebaso (2005), Moriano, Palací y Morales (2006), Lanero, Sánchez, Villanueva y D’Almeida (2007), Tinoco (2008), Lanzas, Lanzas y Lanzas (2009), Salvador (2009), González y Zuñiga (2011), Campos, Figueroa y Sandoval (2011), Merino y Vargas (2011), Lanero, Vázquez, Gutiérrez y García (2011), Moriano, Topa, Molero, Entenza y Lévy-Mangin (2012), Cabana, Cortes, Plaza, Castillo y Álvarez (2013), Rodríguez y Gómez (2014), Alcaraz y Villasana (2015), Pérez y Torralba (2015), Renko, El Tarabishy, Carsrud \& Brännback (2015), entre otros.

Con mucha evidencia de debate y aplicación los trabajos de De Noble et al. (1999), quienes presentan la Escala de Auto-Eficacia Emprendedora (ESE, por sus siglas en inglés), y del mismo equipo de trabajo (Ehrlich et al., 2005), la Escala de Autoeficacia Emprendedora Corporativa (CESE, por sus 
siglas en Inglés); así mismo, sus versiones en castellano, propuestas por Moriano et al (2006) y Moriano et al. (2012), respectivamente.

Por otra parte, autores como Ugalde (2013), quien plantea el capital intelectual como caracterizador del emprendedor, Rodríguez (2015), quién estudia la intensión emprendedora en el ámbito científico público, Cardozo (2010), con el papel de la motivación en el emprendimiento, López (2008), quién evalúa la influencia del emprendedor en el emprendimiento corporativo, y Martínez (2008), quién analiza las competencias emprendedoras en alumnos, entre otros, han tratado el tema a nivel doctoral.

Los resultados de este proyecto de investigación incluyen un perfil de las capacidades de emprendimiento del estudiante de la Unidad Norte de la Universidad de Sonora, y que eventualmente, permitiría el desarrollo de políticas universitarias en el marco del diseño curricular, perfil del egresado, entre otros aspectos. Así mismo, permitiría a la Universidad, contribuir con el desarrollo de políticas gubernamentales.

\section{DISEÑO METODOLÓGICO.}

\subsection{ESTRUCTURA DE LA INVESTIGACIÓN.}

\subsubsection{Objetivo.}

Caracterizar el perfil de la capacidad de emprendimiento de los estudiantes de la Unidad Norte de la Universidad de Sonora revisando el estado del conocimiento en el tema de emprendimiento en México. Valorar un instrumento para medir su capacidad de emprendimiento y así poder identificar los factores que determinan dicha capacidad a través de la determinación de la validez y fiabilidad del instrumento PERFIL DE CAPACIDAD EMPRENDEDORA (PERFIL e20) usado en esta investigación.

\subsubsection{Planteamiento del Problema.}

Para este trabajo se requiere determinar la necesidad de estudiar a fondo las características de emprendimiento de los jóvenes universitarios para promover políticas orientadas al desarrollo que apoyen las actividades productivas, la creación de puestos de trabajo decentes, el emprendimiento, la creatividad y la innovación, y fomentar la formalización y el crecimiento de las microempresas y las pequeñas y medianas empresas, incluso mediante el acceso a servicios financieros.

\subsubsection{Preguntas de investigación}

¿Qué características de emprendimiento poseen los estudiantes universitarios?

¿Cómo se pueden conocer las aptitudes y capacidades de emprendimiento de los estudiantes de la Unidad Norte de la Universidad de Sonora? 


\subsubsection{Hipótesis:}

La capacidad de emprendimiento de los jóvenes estudiantes universitarios es positiva, derivado de la buena formación y calidad de los servicios proporcionados por la Universidad de Sonora en la formación que se les brinda.

\subsubsection{Variables.}

2.1.4.1 Dependiente:

Las capacidades de emprendimiento

\subsubsection{Independiente:}

La calidad de los servicios proporcionados por la Universidad de Sonora en su formación.

\subsubsection{Justificación.}

Durante los últimos años varios investigadores se han hecho interrogantes sobre la orientación y características de la investigación en emprendimiento, como una nueva área del conocimiento que poco a poco cobra mayor importancia en el concierto académico a nivel mundial.

Los resultados de este proyecto de investigación incluyen un perfil de las capacidades de emprendimiento del estudiante de la Universidad de Sonora, y que eventualmente, permitiría el desarrollo de políticas universitarias en el marco del diseño curricular, perfil del egresado, entre otros aspectos. Así mismo, permitiría a la Universidad, contribuir con el desarrollo de políticas gubernamentales.

\subsection{TAMAÑO DE LA MUESTRA Y ESTADÍSTICA DESCRIPTIVA.}

La población objeto del presente estudio se conforma de un universo de estudiantes del 5to. Semestre en adelante de la Universidad de Sonora Unidad Norte, siendo alrededor de 600 estudiantes, de aquí se determinó el tamaño de la muestra de 235 encuestas a alumnos que arrojó el muestreo aleatorio simple, las cuales se repartieron aleatoriamente en la Unidad Regional Norte. Este tamaño de muestra tiene un nivel de confianza del $95 \%$ y un error estimado del 5\%, utilizado para condiciones de validez cuando el estimador del parámetro tiene un comportamiento normal (Barón y Téllez, 2004). Una vez seleccionados los alumnos se procedió a encuestarlos. En este tipo de muestreo todos los sujetos de la población tienen la misma probabilidad de resultar seleccionados. 


\subsection{INSTRUMENTO UTILIZADO.}

En este estudio se ha utilizado una adaptación del instrumento Escala de auto eficacia emprendedora (Entrepreneurial Self-Efficacy, ESE) que proponen Moriano, Palací y Morales, (2006), quienes la adaptaron y validaron. Fue desarrollada en la San Diego State University (SDSU) por los profesores Alex De Noble, Don Jung y Sanford Ehrlich (1999). Estos autores identificaron seis dimensiones que recogían las principales tareas que debe desarrollar un emprendedor para crear con éxito su propia empresa. El instrumento aquí utilizado ha sido el Entrepreneurial Self-Efficacy, ESE, pero complementado con las aportaciones de los diferentes autores mencionados y citados en la revisión literaria de este documento, por lo que ha sido complementado y adaptado al contexto mexicano. El instrumento obtenido ha sido denominado Perfil de Capacidad Emprendedora (PERFIL e20) y consta de 35 ítems principales y 3 ítems globales complementarios para un análisis más profundo. Se espera los resultados se ajusten a las Dimensiones del instrumento adaptado al español, las cuales son las siguientes:

\subsubsection{Factores de la escala ese.}

1) Desarrollar nuevos productos y oportunidades de mercado. Se refiere a un conjunto de habilidades relacionadas con el reconocimiento de oportunidades. El emprendedor debe creer en su capacidad creativa para descubrir oportunidades que le permitan desarrollar sus productos o servicios, y adaptarse a los cambios del mercado (Chell, 2000; Chen et al., 1998; KruegerJR, Reilly y Carsrud, 2000).

2) Construir un entorno innovador. Esta dimensión se centra en la capacidad del individuo para estimular la creatividad, iniciativa y responsabilidad de las personas que trabajan con él. Por lo tanto, el emprendedor debe creer en su capacidad para construir un entorno a partir de cero que favorezca la innovación. Este factor de la escala ESE está relacionado con la dimensión "asunción de riesgos e innovación" hallada por Chen et al. (1998).

3) Iniciar relaciones con inversores. Los emprendedores deben utilizar sus redes sociales y establecer contactos que les permitan captar los recursos necesarios para crear su propia empresa (Ehrlich, De Noble, Moore y Weaver, 1994).

4) Definir el objetivo central del negocio. Esta dimensión es fundamental porque si una persona se cree incapaz de establecer el propósito principal de su negocio, entonces resulta poco probable que se sienta motivado para iniciar su propia aventura empresarial.

5) Afrontar cambios inesperados. Se refiere a la creencia sobre la capacidad de trabajar bajo incertidumbre. Adentrarse en el mundo de la creación de empresas, dejando atrás el confort que supone trabajar por cuenta ajena en una empresa establecida, requiere una tolerancia a la ambigüedad y adaptación a los cambios. 
6) Desarrollar los recursos humanos clave. Habilidad para atraer y retener individuos que son claves en la creación de una nueva empresa. Además, resulta básico que el emprendedor reconozca la importancia de involucrar a otros en el proceso de creación de su empresa.

En la sección I del instrumento se utiliza un formato de dos columnas: La primera contiene los ítems con las características de emprendimiento; la segunda dedicada para determinar el grado de cumplimiento con cada ítem. En la sección II se utiliza el mismo formato de dos columnas y se enfoca en la seguridad, factibilidad e intención emprendedora.

Al analizar los resultados, cada una de sus dimensiones son consideradas como variables continuas, y las puntuaciones de los sujetos son clasificadas mediante un sistema de valores para cada escala (Maneiro, Mejías, Romero y Serpa, 2008), (Tabla 1).

En el presente trabajo se ha aplicado una escala de Likert de cinco posibilidades de respuesta (de 1 a 5) por lo que los anteriores rangos para evaluar el nivel de las dimensiones han sido recalculados para este tipo de escala, respetando el sistema de percentiles para la Escala Likert propuesto por Maneiro, Mejías, Romero y Serpa, (2008) y replanteado por Vega-Robles et al. (2014).

Para este caso, los promedios de los valores del instrumento que caigan en valores menores a 1,79 se consideran Totalmente de Acuerdo. Los promedios mayores a 1,79 y menores o iguales a 2,59 se les consideran De Acuerdo. A los promedios que caigan entre 2,591 y 3,39 se les considera ni De acuerdo Ni en Desacuerdo, a los promedios de las dimensiones que caigan entre 3,391 y 4,19 se les considera De Acuerdo y, por último, a los promedios que sobrepasen a 4,21 hasta 5, se les considera como un Completamente De Acuerdo de poder ser emprendedores.

Para determinar el nivel de satisfacción del cliente a través del servicio recibido, se presenta la tabla referente a esta escala (ver Tabla 1).

Tabla 1.- Puntuación Likert para las encuestas aplicadas a los estudiantes.
\begin{tabular}{l|l|l} 
Escala de Likert & $\begin{array}{l}\% \\
\text { Cumplimiento. }\end{array}$ \\
\hline 1,00 a 1,79 & Totalmente en Desacuerdo & 20,0 a 35,9 \\
\hline 1,80 a 2,59 & En Desacuerdo & 36,0 a 51,9 \\
\hline 2,60 a 3,39 & $\begin{array}{l}\text { Ni en desacuerdo Ni de } \\
\text { Acuerdo }\end{array}$ & 52,0 a 67,9 \\
\hline 3,40 a 4,19 & De Acuerdo & 68,0 a 83,9 \\
\hline 4,20 a 5,00 & Completamente de Acuerdo & 84,0 a 100 \\
\hline
\end{tabular}

Fuente: Maneiro, Mejías, Romero y Zerpa, (2008).

\subsubsection{Validación del Instrumento de Medición.}

De Noble et al. (1999), presentan la Escala de Auto-Eficacia Emprendedora (ESE, por sus siglas en inglés), así mismo, su versión en castellano, fue propuesta por Moriano et al. (2006). Todos ellos, junto 
con Martínez (2008), quién analiza las competencias emprendedoras en alumnos, entre otros, han tratado el tema a nivel doctoral.

Para utilizar este instrumento de Características de emprendimiento, primero es necesario corroborar la fiabilidad del instrumento en forma global.

El objetivo del análisis de fiabilidad es determinar que un conjunto de elementos (ítems) de una escala, puedan conducir a resultados que estén altamente correlacionados con los resultados que se llegarían a obtener si se repitiera la prueba. Es decir, consiste en lograr una escala que conduzca a resultados similares cuando diferentes personas la administren y cuando usan formas alternas de la prueba (Merino y Lautenschlager, 2003).

Para determinar la fiabilidad hay diferentes formas de hacerlo, la más común es utilizar el Alpha de Cronbach, que está orientada hacia la consistencia interna de una prueba, para valores inferiores a 0,6 se considera una baja fiabilidad, entre 0,6 y 0,8 es aceptable y por encima de 0,8 es excelente (Caetano y Nuno, 2003).

Según Carretero y Pérez (2005) el coeficiente Alpha de Cronbach es directamente proporcional al número de preguntas, esto significa que se incrementa cuanto mayor sea el número de ítems consideradas. Es por esto, que, al someter al análisis de fiabilidad a cada una de las seis dimensiones, los coeficientes suelen dar debajo de 0,8 , lo cual los hace ver como que no son suficientemente válidos, donde ya se ve la validez es cuando se consideran en forma global las 35 preguntas.

Una vez aplicado el instrumento Perfil de la Capacidad Emprendedora es necesario realizar evaluaciones periódicas para saber si las capacidades emprendedoras responden efectivamente a las necesidades de formación de los estudiantes, al mismo tiempo durante este proceso se pueden identificar las debilidades, oportunidades, fortalezas y amenazas en la formación académica y profesional de estos estudiantes. La importancia de este estudio es que la información que sea generada puede permitir a la Universidad de Sonora considerar el desarrollo de políticas universitarias en el marco del diseño curricular, perfil del egresado, entre otros aspectos. Así mismo, permitiría a la Universidad, contribuir con el desarrollo de políticas gubernamentales y además esta investigación generará conocimiento al comparar con otras instituciones de otras partes de México.

\subsection{PROCEDIMIENTO.}

El análisis de los datos se efectuó mediante el paquete estadístico SPSS 23 para Windows. Se empleó el método de Componentes Principales con rotación Varimax para la extracción de factores, y se retuvieron aquellos factores con eigenvalue mayor que 1 (Kaiser, 1960; 
Tabachnik y Fidell, 2007). Antes de efectuar el análisis factorial (AF), se revisó el cumplimiento de ciertos criterios para cumplir con la viabilidad del mismo.

El determinante de la matriz de correlaciones obtuvo un valor de 0,0000001744. La prueba de esfericidad de Bartlett fue significativa, el test KMO de adecuación de la muestra arrojó un valor de 0,917, la fiabilidad del instrumento fue de 0,942 y la prueba detallada de Normalidad de Kolmovorov-Smirnov fue significativa para todos los ítems. Estos resultados indican que es válido efectuar un análisis factorial de la matriz de correlaciones (Tabachnick y Fidell, 2007; Hair et al. 2010) y que el instrumento es muy confiable. Hair et al. (2010) sugiere que para que una carga factorial pueda ser considerada como significativa su valor no debe ser inferior a 0,45 (esto equivale a $\mathrm{n}=150$ ). Por otro lado, Morales (2011) sugiere que una carga factorial de 0,25 (equivalente a $n=400$ ) ya se puede considerar como significativa, aunque ambos reconocen que depende mucho de la experiencia empírica del investigador y del sustento teórico del constructo. En el caso de este estudio como $n=235$, entonces el punto de corte de significancia para una carga factorial es igual o mayor a 0,38. Para lograr una mayor consistencia, se escogió a partir de 0,4 como cargas significativas.

\section{RESULTADOS}

En el presente estudio el nivel de confiabilidad de Alfa de Cronbach igual a 0,942 la cual se considera como muy aceptable en estudios referentes a las ciencias sociales. Con este indicador, la aplicación del instrumento muestra muy buen nivel de confiabilidad del mismo.

$\mathrm{El}$ análisis factorial (AF) se ha aplicado para presentar los resultados obtenidos para proveer un sustento cuantitativo que permita obtener una medida objetiva de las características de emprendimiento de los estudiantes e identificar los factores que pueden ser considerados más importantes a la hora de analizar dichas características. Dicho análisis se ha realizado utilizando la técnica de análisis de componentes principales, con la que se han sintetizado los datos pudiéndolos relacionar entre sí, obteniéndose las características principales que definen la estructura dimensional del constructo que deben de ser incluidas en el análisis de medición (ver Tabla 2).

El AF arrojó un arreglo de 8 factores, pero para acercar más este resultado a las dimensiones teóricas de los autores de este instrumento de medición, los autores del presente trabajo en un afán por acercar más el arreglo factorial obtenido al arreglo dimensional teórico del constructo de seis Dimensiones ya descritas en el presente trabajo, han optado por repetir el análisis factorial para 7 y para 6 factores para estudiar la variación de los parámetros estadísticos y aplicar el Principio de Parsimonia (López y Baniandrés, (2013)), buscando obtener un modelo más sencillo, con menos Dimensiones pero que también 
represente fielmente a los datos analizados. Los resultados obtenidos se resumen en la Tabla 2 y el arreglo factorial obtenido se presenta en la Tabla 3.

Tabla 2.- Resultados de los diferentes Análisis Factoriales realizados.

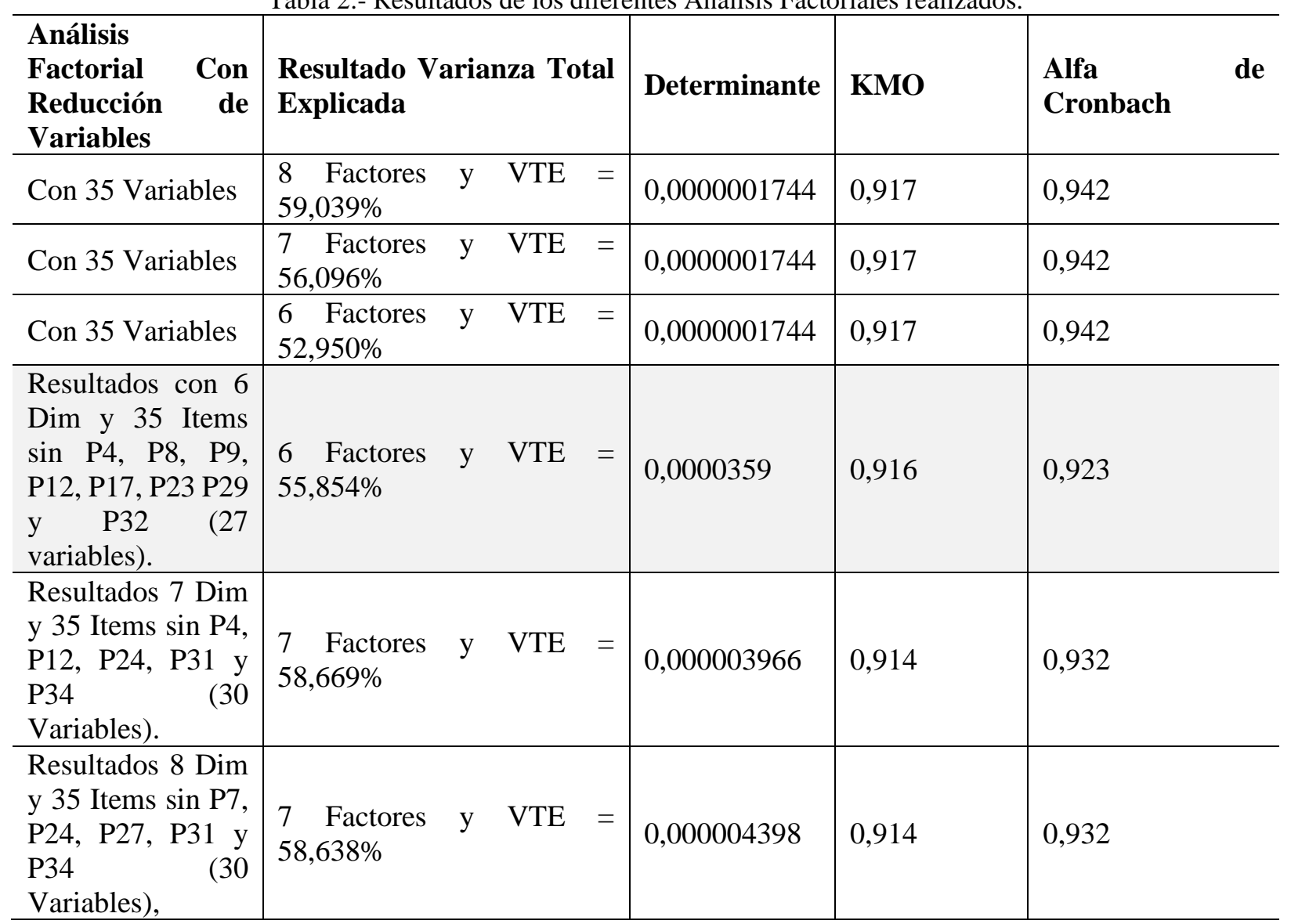

Fuente: Elaboración propia con resultados de SPSS 23.

Tabla 3. Matriz de componentes $\operatorname{rotados}^{\mathrm{a}}$

\begin{tabular}{|c|c|c|c|c|c|c|}
\hline & \multicolumn{6}{|c|}{ Componentes } \\
\hline & 1 & 2 & 3 & 4 & 5 & 6 \\
\hline $\mathrm{P} 15$ & 0,642 & & & & & \\
\hline P7 & 0,594 & & & & & \\
\hline P31 & 0,563 & & & & & \\
\hline $\mathrm{P} 30$ & 0,539 & & & & & \\
\hline P6 & 0,532 & & & & & \\
\hline $\mathrm{P} 14$ & 0,523 & & & & & \\
\hline P35 & 0,430 & & & & & \\
\hline $\mathrm{P} 20$ & & 0,752 & & & & \\
\hline P26 & & 0,631 & & & & \\
\hline P24 & & 0,504 & & & & \\
\hline P33 & & 0,408 & & & & \\
\hline $\mathrm{P} 22$ & & 0,375 & & & & \\
\hline P19 & & & 0,710 & & & \\
\hline $\mathrm{P} 13$ & & & 0,635 & & & \\
\hline $\mathrm{P} 11$ & & & 0,507 & & & \\
\hline P27 & & & 0,456 & & & \\
\hline $\mathrm{P} 25$ & & & & 0,695 & & \\
\hline $\mathrm{P} 21$ & & & & 0,518 & & \\
\hline
\end{tabular}




\begin{tabular}{|c|c|c|c|}
\hline $\mathrm{P} 10$ & 0,503 & & \\
\hline P34 & 0,484 & & \\
\hline $\mathrm{P} 16$ & & 0,701 & \\
\hline $\mathrm{P} 5$ & & 0,566 & \\
\hline P3 & & 0,511 & \\
\hline $\mathrm{P} 1$ & & 0,467 & \\
\hline $\mathrm{P} 28$ & & & 0,700 \\
\hline $\mathrm{P} 2$ & & & 0,463 \\
\hline P18 & & & 0,397 \\
\hline
\end{tabular}

Método de extracción: Análisis de componentes principales. Método de rotación: Normalización Varimax con Kaiser. a. La rotación ha convergido en 31 iteraciones.

Fuente: Obtenida de SPSS 23.

Al obtener el arreglo factorial inicial con ocho factores se pudo observar que las variables P7, P24, P27, P31 y P34 resultaron ambiguas ya que sus autovalores eran muy cercanos en más de un Factor con diferencias menores a 0,1 por lo que fueron eliminadas dichas variables, volviéndose a efectuar el análisis factorial, pero ahora para las 30 variables restantes. Del AF de siete factores se observaron que las variables P4, P12, P24, P31 y P34 también resultaban ambiguas por la misma razón ya descrita y del AF para seis factores se observó también ambigüedad en las variables P4, P8, P9, P12, P17, P23, P29 y P32 por lo que se eliminaron dichas variables, efectuándose de nuevo el AF para ambos casos con 30 y con 27 variables respectivamente. Los resultados de todos los casos se presentan en la Tabla 2. El arreglo factorial para seis factores se presenta en la Tabla 3. Por el Principio de Parsimonia ya explicado antes y buscando una mayor simplicidad en el arreglo obtenido, se escogió como resultado aceptable el arreglo factorial de seis factores y 27 variables, ya que las diferencias con el arreglo original de ocho factores y 35 variables no son significativas: el valor del Determinante sigue siendo muy cercano a cero, la Varianza Total Explicada solo cae del 59,039\% al 55,854\%, una disminución de tan solo el 3,18\% , El valor de KMO solo disminuye de 0,917 a 0,916 y el alfa de Cronbach solo disminuye de 0,942 a 0,923, siendo estos, valores muy altos y adecuados para un análisis de este tipo. (Kaiser, 1960; Tabachnik y Fidell, 2007; Hair et al. 2010).

Como Resultado del Análisis Factorial aceptado se obtuvo el arreglo de los Factores que Determinan la Capacidad de Emprendimiento en un Estudiante. Ver Tabla 4.

Tabla 4. Factores que determinan la Capacidad de Emprendimiento en un Estudiante.

\begin{tabular}{ll}
\hline P15 & Me gusta alcanzar los objetivos que me propongo \\
\hline P7 & Tengo confianza en mis capacidades y posibilidades \\
\hline P31 & Suelo cumplir los compromisos para acabar un trabajo \\
\hline P30 & Me considero bueno en el trabajo que hago \\
\hline P6 & Me gusta asumir responsabilidades \\
\hline P14 & Me gusta tener capacidad de decisión \\
\hline P35 & Sueles cumplir los plazos que te fijas para realizar un trabajo \\
\hline P20 & Me considero una persona ambiciosa \\
\hline P26 & Me considero una persona motivada \\
\hline
\end{tabular}




\begin{tabular}{ll}
\hline P24 & Me gustan los retos \\
\hline P33 & Siento mi espíritu aventurero \\
\hline P22 & Se encontrar soluciones ante los problemas \\
\hline P19 & Me resulta fácil asignar tareas a los demás \\
\hline P13 & Si necesito ayuda, la pido \\
\hline P11 & Para mí es importante disponer de autonomía en mi trabajo \\
\hline P27 & Si el trabajo lo requiere, soy capaz de sacrificar mi tiempo libre \\
\hline P25 & Se me da bien administrar los recursos económicos \\
\hline P21 & Tengo predisposición para asumir riesgos \\
\hline P10 & Me considero una persona ambiciosa \\
\hline P34 & Si existe algo que "no hay manera de hacer", encuentro esa manera \\
\hline P16 & Me considero una persona creativa \\
\hline P5 & Soy una persona intuitiva \\
\hline P3 & Me adapto con facilidad a los cambios \\
\hline P1 & Me considero una persona emprendedora \\
\hline P28 & Tengo facilidad de comunicación \\
\hline P2 & Soy una persona con una actitud positiva \\
\hline P18 & Me considero una persona optimista \\
\hline
\end{tabular}

\section{CONCLUSIONES Y DISCUSIÓN.}

Se aplicó el instrumento PERFIL DE CAPACIDAD EMPRENDEDORA (PERFIL e20) a una muestra de 235 alumnos del 5to. Semestre en adelante de la Universidad de Sonora Unidad Norte. Se efectuaron las pruebas requeridas para considerar válido un análisis factorial resultando válidas estas pruebas con un determinante de 0,0000001744, La prueba de esfericidad de Bartlett fue significativa, el test KMO de adecuación de la muestra fue de 0,917, la fiabilidad del instrumento fue de 0,942 y la prueba de Normalidad de Kolmovorov-Smirnov fue significativa para todos los ítems y el nivel de confiabilidad de Alfa de Cronbach igual a 0,942. En el caso de este estudio como $n=235$, entonces el punto de corte de significancia para una carga factorial significativa es igual o mayor a 0,38.

El análisis factorial arrojó un arreglo de ocho factores que se aparta de la teoría del constructo de este instrumento para medir el Emprendimiento. Se repitió este análisis para obtener siete y seis factores y así obtener valores y poder realizar un comparativo respecto a la Varianza Total Explicada (VTE), su Determinante, su KMO y su Alfa de Cronbach. Al analizar el resultado con seis dimensiones se pudo ver que las variables P4, P8, P9, P12, P17, P23, P28 y P32 resultaban muy ambiguas ya que sus autovalores caían en más de un factor por lo que fueron eliminadas, atendiendo el Principio de Parsimonia y buscando obtener simplicidad de descripción y cálculo. Disminuyó el número de variables de 35 a 27. Se escogió este arreglo factorial ya que su VTE fue de 55,854\%, el valor del Determinante 0000,359, KMO=0,916 y con una confiabilidad de 0,923 ; todos estos valores considerados muy buenos para la experimentación.

Como resultado del Análisis Factorial para seis factores se obtuvo el arreglo de las Dimensiones de este Constructo el cual se presenta a continuación en la tabla 5. 
Tabla 5.- Dimensiones Obtenidas del Análisis Factorial.

\begin{tabular}{|c|c|c|}
\hline \multicolumn{3}{|c|}{$\begin{array}{l}\text { DIMENSIÓN 1: DESARROLLAR NUEVOS PRODUCTOS Y OPORTUNIDADES DE } \\
\text { MERCADO. }\end{array}$} \\
\hline & & P15 $\quad$ Me gusta alcanzar los objetivos que me propongo \\
\hline & & Tengo confianza en mis capacidades y posibilidades \\
\hline & & Suelo cumplir los compromisos para acabar un trabajo \\
\hline & & Me considero bueno en el trabajo que hago \\
\hline & & Me gusta asumir responsabilidades \\
\hline & & Me gusta tener capacidad de decisión \\
\hline & & Suelo cumplir los plazos que te fijas para realizar un trabajo \\
\hline \multicolumn{3}{|c|}{ DIMENSIÓN 2: CONSTRUIR UN ENTORNO INNOVADOR. } \\
\hline & & Me considero una persona creativa \\
\hline & & Soy una persona intuitiva \\
\hline & & Me adapto con facilidad a los cambios \\
\hline & & Me considero una persona emprendedora \\
\hline \multicolumn{3}{|c|}{ DIMENSIÓN 3: INICIAR RELACIONES CON INVERSORES. } \\
\hline & & Se me da bien administrar los recursos económicos \\
\hline & & Tengo predisposición para asumir riesgos \\
\hline & & Me considero una persona ambiciosa \\
\hline & & Si existe algo que "no hay manera de hacer", encuentro esa manera \\
\hline \multicolumn{3}{|r|}{ DIMENSIÓN 4: DEFINIR EL OBJETIVO CENTRAL DEL NEGOCIO. } \\
\hline & & Tengo facilidad de comunicación \\
\hline & & Soy una persona con una actitud positiva \\
\hline & & Me considero una persona optimista \\
\hline \multicolumn{3}{|c|}{ DIMENSIÓN 5: AFRONTAR CAMBIOS INESPERADOS. } \\
\hline & & Me considero una persona ambiciosa \\
\hline & & Me considero una persona motivada \\
\hline & & Me gustan los retos \\
\hline & & Siento mi espíritu aventurero \\
\hline & & Se encontrar soluciones ante los problemas \\
\hline \multicolumn{3}{|r|}{ DIMENSIÓN 6: DESARROLLAR LOS RECURSOS HUMANOS CLAVE. } \\
\hline & & Me resulta fácil asignar tareas a los demás \\
\hline & & Si necesito ayuda, la pido \\
\hline & & Para mí es importante disponer de autonomía en mi trabajo \\
\hline & & Si el trabajo lo requiere, soy capaz de sacrificar mi tiempo libre \\
\hline
\end{tabular}

Fuente: Elaboración propia con resultados de SPSS.

En la Tabla 5 se pueden identificar las dimensiones y variables que caracterizan el perfil de emprendimiento de los estudiantes de la Unidad Norte de la Universidad de Sonora. Esta información su puede utilizar para facilitar la toma de decisiones en el diseño de estrategias curriculares y de enseñanzaaprendizaje, perfil de egresado; entre otros aspectos. También permite el desarrollo de políticas universitarias que contribuyan con el desarrollo de políticas gubernamentales en apoyo a los jóvenes emprendedores. 


\section{REFERENCIAS}

Alcaráz, R. y Vilasana, M. (2015). Construcción y validación de un instrumento para medir competencias emprendedoras. XIX Congreso Internacional de Investigación en Ciencias Administrativas: Gestión de las Organizaciones rumbo al 3er milenio "de la Regionalización a la Globalización", pp. 1-31. Academia de Ciencias Administrativas ACACIA, México.

Bruyat, C. and Julien, P.A. (2000). Defining the Field of Research in Entrepreneurship. Journal of Business Venturing, Vol. 16, No.2, pp.165-80.

Busenitz, L., West, G., Shepherd, D., Nelson, T., Chandler, G., and Zacharakis, A. (2003). Entrepreneurship Research in Emergence: Past Trends and Future Directions. Journal of Management. 29(3) 285-308. DOI: 10.1016/S0149-2063_03_00013-8.

Cabana-Villca, R.; Cortes-Castillo, I.; Plaza-Pasten, D.; Castillo-Vergara, M. y Alvarez-Marin, A. (2013). Análisis de Las Capacidades Emprendedoras Potenciales y Efectivas en Alumnos de Centros de Educación Superior. Journal of Technology, Management \& Innovation, Vol. 8, No. 1, pp. 65-75.

Caetano, A. y Gonzalo, N. (2003). Marketing en los servicios de educación modelos de percepción de calidad. Tesis Doctoral Universidad Complutense de Madrid, Facultad de Ciencias Económicas y Empresariales, Departamento de Comercialización e Investigación de Mercados.

Campos, R.; Figueroa, G. y Sandoval, M. (2011). Medición de las habilidades emprendedoras: base para mejorar el programa de desarrollo emprendedor. XV Congreso internacional sobre innovaciones en docencia e investigación en ciencias económico administrativas. Chihuahua, Chihuahua, 5, 6 y 7 de septiembre de 2012.

Cardozo, A. (2010). La motivación para emprender. Evolución del modelo de rol en emprendedores argentinos. Tesis Doctoral. Universidad Nacional de Educación a Distancia, España.

Carretero-Dios, H. Pérez, C. (2005). Normas para el desarrollo y revisión de estudios instrumentales. International Journal of Clinical and Health Psychology. Vol. 5, No. 3, pp. 521-551 [Fecha de consulta: 31 de julio de 2019] Disponible en: http://www.redalyc.org/articulo.oa?id=33705307 ISSN 1697-2600.

Castillo, A. (1999). Estado del arte en la enseñanza del emprendimiento. Programa

Emprendedores como creadores de riqueza y desarrollo regional Intec. Chile: Intec.

Chell, E. 2000. "Towards researching the "opportunistic entrepreneur": A social constructionist approach and research agenda." European Journal of Work \& Organizational Psychology 9(1):63-80.

Chen, C., Green, P. and Crick, A. (1998). Does Entrepreneural Self-efficacy Distinguish Entrepreneurs from Managers? Journal of Business Venturing, 13, 295-316.

Cooper, D. R., \& Schindler, P. S. (2003). Business research methods. 8th ed. Boston, Mass.: McGrawHill/Irwin.

Denoble, A., Jung, D. \& Ehrlich, S. (1999). Entrepreneurial self-efficacy: The development of a measure and its relationship to entrepre-neurial actions. Trabajo presentado en el Frontiers of Entrepreneurship Research, Waltham. 
Ehrlich, S., De Noble, A., Moore, T. y Weaver, R. (1994). After the cash arrives: A comparative study of venture capital and private investor involvement in entreprenurial firms. Journal of Business Venturing, $9,67-82$.

Ehrlich, S., De Noble, A. y Singh, J. (2005). Corporate Entrepreneurial Self-Efficacy: Toward the Development of a Domain-Specific Measure'.Paper pre-sented at the Frontiers of Entrepreneurship Research, Babson.

Fernández, A. (2015). Informe GEM España 2014. España: Universidad de Cantabria.

González, R. y Zúñiga, A. (2011). Método CEPCES para la Evaluación del Potencial Emprendedor. Journal of Technology, Management \& Innovation, Vol. 7, No. 1, pp. 77-99.

Guzmán V., y Trujillo, D. (2008). Emprendimiento social - revisión de literatura. Estudios gerenciales. 24 (109), 105-125.

Hair, J., Anderson, R., Tatham, R. y Black, W. (2010). Análisis Multivariante. 5ta. Ed. Madrid: Ed. Pearson Prentice-Hall.

Herrera, C. y Montoya, L. (2013). El emprendedor: una aproximación a su definición y caracterización. Punto de vista, Vol. 4, No. 7, pp. 7-30.

Hidalgo, G.; Kamiya, M. y Reyes, M. (2014). Emprendimientos dinámicos en América Latina. Avances en prácticas y políticas. Serie Políticas Públicas y Transformación Productiva, N¹6. Corporación Andina de Fomento / Banco de Desarrollo de América Latina.

Hisrich, R., Peters, M., \& Shepherd, D. (2005). Entrepreneurship (Sexta ed.). México: Mc Graw Hill 2005. ISBN: 9780072873740

Hisrich, R., Langan-Fox J., \& Grant, S. (2007). Entrepreneurship Research and Practice: A Call to Action for Psychology. American Psychologist. Vol. 62, No. 6. pp. 575-589. DOI: 10.1037/0003-066X.62.6.575.

Kaiser, H. (1960). The application of electronic computers to analysis factorial. Educational and Psychological Measurement, 20, 141-151.

Karmarkar, Y., Chabra, M., and Desphande, A. (2014). Entrepreneurial Leadership Style(s): A Taxonomic Review. Annual Research Journal of Symbiosis Centre for Management Studies. 2(1), 156-189.

Krueger Jr, N., Reilly, M., \& Carsrud, A. (2000). Competing models of entrepreneurial intentions. Journal of business Venturing, 15(5), 411-432.

Lanero, A., Sánchez, J., Villanueva, J., y D’Almeida, M. (2007). La perspectiva cognitiva en el proceso emprendedor. Guillén C. \& R. Guil (Coords.), X Congreso Nacional de Psicología Social: Un Encuentro de Perspectivas (Vol. 2, pp. 1594-1604). Cádiz, Spain: University of Cádiz.

Lanero, A.; Vázquez, J.; Gutiérrez, P. y García, M. [2011]. Evaluación de la conducta emprendedora en estudiantes universitarios. Implicaciones para el diseño de programas académicos. Pecvnia, No. 12, pp. 219-243. 
Lanzas, V.; Lanzas, F. y Lanzas, A. (2009). Propuesta para medir el perfil de los emprendedores de base tecnológica. Scientia et Technica, Año XV, No. 43, pp. 267-272.

Leitch, C., \& Volery, T. (2017). Entrepreneurial leadership: Insights and directions. International Small Business Journal, 35 (2), 147-156.

Leiva, J. (2008). ¿Nacen empresas de las actividades de fomento al espíritu emprendedor?: un vistazo a empresas surgidas del Concurso Nacional de Emprendedores y el Programa de Formación en Espíritu Emprendedor del Instituto Tecnológico de Costa Rica. Tec Empresarial, ISSN-e 1659-3359, Vol. 2, Nº. 1, 2008, pp. 16-27.

López, C. (2008). Influencia del comportamiento emprendedor de los mandos medios en el fomento del Corporate Entrepreneurship. Tesis Doctoral. Universitat Ramon Llull, España.

López, C., y Baniandrés, N. (2013). El principio de parsimonia en la ciencia cognitiva actual: Riesgos y soluciones. Ciencia Cognitiva, 7:2, 28-30. Recuperado de http://www.cienciacognitiva.org/files/201310.pdf

Low, M. (2001). The Adolescence of Entrepreneurship Research: Specification of Purpose. Columbia Business School. Volume: 25 issue: 4, page(s): 17-26. https://doi.org/10.1177/104225870102500402

Maneiro, N.; Mejías, M.; Romero, M. y Zerpa, J. (2008) Evaluación de la Calidad de los Servicios, una Experiencia en la Educación Superior Venezolana. EDUCERE, Vol. 12, No. 43, pp. 797-804.

Matiz, F. (2009). Investigación en emprendimiento, un reto para la construcción de conocimiento. Revista EAN, No. 66, pp. 169-182.

Martínez, F. (2008). Análisis de competencias emprendedoras del alumnado de las escuelas taller y casa de oficio en Andalucía. Primera fase del diseño de programas educativos para el desarrollo de la cultura emprendedora entre los jóvenes. Tesis Doctoral. Universidad de Granada, España.

Merino, César. \& Lautenschlager, Gary. (2003). Comparación estadística de la confiabilidad alfa de Cronbach: Aplicaciones en la medición educacional y psicológica. Revista de Psicología - Universidad de Chile, 12(2), 129 - 139.

Merino, M. y Vargas, D. (2011). Evaluación comparativa del potencial emprendedor de Latinoamérica: una perspectiva multinivel. Academia, Revista Latinoamericana de Administración, No. 46, pp. 38-54.

Moriano, J.; Sánchez, M. y Palací, F. (2004). Un estudio descriptivo sobre los emprendedores en España, la República Checa y Bulgaria (cap. 10). En "Congreso El emprendedor innovador y la creación de empresas de I+D+I”, capitulo 10, pp. 161-178. Universidad de Valencia: Valencia, España.

Moriano, J.; Palací, F. y Morales, J. (2006). Adaptación y validación en España de la escala de Autoeficacia Emprendedora. Revista de Psicología Social, Vol. 21, No.1, pp. 51-64.

Moriano, J.; Topa, G.; Molero, F.; Entenza, A. y Lévy-Mangin, J. (2012). Autoeficacia para el Liderazgo Emprendedor. Adaptación y Validación de la Escala CESE en España. Anales de psicología, Vol. 28, No. 1, pp. 171-179. 
Murillo, E. y Santillán, L. (2015). Revisión de las Investigaciones sobre teoría y práctica del Emprendimiento. Posibilidades del Monitor Global de Emprendimiento (MGE). Revista Publicando, Vol. 2, No. 5, pp. 285-299.

Naciones Unidas (2016). Agenda 2030 y los Objetivos de Desarrollo Sostenible. Una oportunidad para América Latina y el Caribe. Santiago. CEPAL/UN.

Nicolás, C. y Rubio, A. (2012). El emprendimiento social: una comparativa entre España y países sudamericanos. Revista FIR, FAEDPYME International Review, Vol. 1, No. 1, pp. 38-49.

Pérez, A y Torralba, A. (2015). Medición del emprendedurismo en el municipio de Puebla: Diagnóstico para el desarrollo empresarial. Tec Empresarial, Vol. 9, No. 1, pp. 19-30.

Renko, M., El Tarabishy, A., Carsrud, A., Brännback, M. (2013). Understanding and measuring entrepreneurial leadership style. Journal of Small Business Management. Vol.53, No. 1. pp 54-74. https://doi.org/10.1111/jsbm.12086

Rodríguez, D. y Gómez, A. (2014). Las competencias emprendedoras en el departamento de Boyacá. Apuntes del CENES, Vol. 33, No. 58, pp. 217-242.

Rodríguez, F. (2015). Estudio de la intensión emprendedora en el ámbito científico público. El caso de las ciencias de la vida en España. Tesis Doctoral. Universidad de Valencia, España.

Salvador, C. (2009). Ecuaciones estructurales como modelos predictivos de la autoeficacia emprendedora en una muestra de jóvenes mexicanos y españoles. Apuntes de Psicología, Vol. 27, número 1, pp. 65-78.

Sánchez, J.; Lanero, J. y Yurrebaso, A. (2005). Variables determinantes de la intención emprendedora en el contexto universitario. Revista de Psicología Social Aplicada, Vol. 15, nº 1, pp. 37-60.

Shane, S. y Venkataraman, S. (2000). The Promise of Entrepreneurship as a Field of Research. Academy of Management Review, 25 (1), 217-226.

Tabachnick, B. y Fidell, L. (2007). Using multivariate statistics. 5th Edition. Boston: Harper Collins.

Tinoco, O. (2008). Medición de la Capacidad Emprendedora de ingresantes a la Facultad de Ingeniería Industrial de la UNMSM. Revista de la Facultad de Ingeniería Industrial, Vol. 11, No. 2, pp 18-23.

Ugalde, N. (2013). Capital intelectual, características del emprendedor e innovación. El caso de las MIPYMES Costarricenses. Tesis Doctoral. Universidad de Valencia, España.

VECIANA, J. (1999) “Creación de Empresas como programa de investigación Científica” Revista Europea de Dirección y Economía de la Empresa, Vol. 8, No. 3. pp 11-36.

Vega, R., Cadena, J., Mejías., A, y Guzmán, R. (2014). Análisis de la calidad de los servicios académicos: caso de estudio Ingeniería Industrial y de Sistemas Campus Caborca, Universidad de Sonora, México. Memorias del VII Simposio Internacional de Ingeniería Industrial: Actualidad y Nuevas Tendencias 2014. Universidad Nacional Mayor de San Marcos Lima, Perú 2014. ISSN 1856-8343. 
Wiklund, J., Davidsson, P., Audretsch, D. \& Karlsson, C. (2011). The Future of Entrepreneurship Research. Entrepreneurship Theory and Practice. Volume: 35 issue: 1, page(s): 1-9. https://doi.org/10.1111/j.1540-6520.2010.00420.x

Zahra, S., Sapienza, H. \& Davidsson, P. (2006) 'Entrepreneurship and dynamic capabilities: a review, model and research agenda', Journal of Management Studies, 43: 917-955. 